\title{
The Occurrence of Antibiotic Resistant Bacteria Contamination in Sub-district Health-promoting Hospitals in Chiang Rai, Thailand
}

\author{
Korakot Chansareewittaya, Ph.D. ${ }^{1}$, Sirikarnnapa Krajangcharoensakul, B.Sc. ${ }^{2}$ \\ ${ }^{1}$ Public Health Major, School of Health Science, Mae Fah Luang University, Mueang, Chiang Rai 57100, Thailand. \\ ${ }^{2}$ Scientific and Technological Instruments Center, Mae Fah Luang University, Mueang, Chiang Rai 57100, Thailand. \\ Received 30 August 2021 • Revised 15 October 2021 • Accepted 19 October 2021 • Published online 22 December 2021
}

\begin{abstract}
:
Objective: To determine the antibiotic susceptibility patterns (antibiogram profiles) of the bacterial agents usually involved in hospital-acquired infections found in 12 sub-district health-promoting hospitals (HPHs) in Chiang Rai, Thailand.

Material and Methods: Swabs from 10 different sampling points in each sub-district HPH were aseptically collected. Standard microbiological methods were performed to define the bacterial species. Antibiotic susceptibility was determined by the disk diffusion method following the standard guidelines of the Clinical and Laboratory Standards Institute (2016). Results: The antibiogram profiles of the 153 isolated bacteria showed that $55.6 \%$ of the isolates were resistant to antibiotics. Single drug resistant, double drug resistant, and multi-drug resistant bacteria accounted for 18.3\%, 18.3\%, and 19.0\%, respectively. The Pseudomonas aeruginosa isolate was susceptible to all tested antibiotics. MDR phenotypes were most common in coagulase-negative staphylococci (13.1\%), followed by members of the family of Enterobacteriaceae (3.9\%) and Staphylococcus aureus $(0.7 \%)$.

Conclusion: The MDR rates reported in this study are "worrying". These results suggest that sub-district HPHs may become sources of HAls caused by antibiotic-resistant bacteria which can be inevitably transmitted into the wider community. Antibiotic stewardship, antibiotic susceptibility surveillance and hygiene practices may be used to prevent and limit the spread of such bacteria from sub-district HPHs to the community.
\end{abstract}

Keywords: antibiogram profiles, antibiotic resistant bacteria, sub-district health-promoting hospitals

Contact: Korakot Chansareewittaya, Ph.D.

Public Health Major, School of Health Science, Mae Fah Luang University,

Mueang, Chiang Rai 57100, Thailand.

E-mail: korakot.cha@mfu.ac.th

() 2021 JHSMR. Hosting by Prince of Songkla University. All rights reserved.

This is an open access article under the CC BY-NC-ND license

(http://www.jhsmr.org/index.php/jhsmr/about/editorialPolicies\#openAccessPolicy).
J Health Sci Med Res 2022;40(4):459-473 doi: 10.31584 /jhsmr.2021857 www.jhsmr.org 


\section{Introduction}

Bacterial infections in hospital environments are known as hospital-acquired infections (HAls) or nosocomial infections. The cross-transmission of $\mathrm{HAl}$ pathogens occurs via patient-to-patient contact or contact with patientenvironments and hospital personnel-patient routes, as a result of hospital contamination and poor hygiene. ${ }^{1}$ Several studies have reported that the improper use of antibiotic therapies promote the presence of antibiotic-resistant bacteria (ARB) within hospital settings ${ }^{2,3}$, although $\mathrm{HAl}$ transmission of ARB in hospitals is not typical of epidemic models. ${ }^{4}$ This was supported by a recent study by Cai et al. ${ }^{5}$ which revealed that the abundance and types of $A R B$ as well as their particular antibiotic-resistant genes were significantly determined by the extensive use of antibiotics in hospitals. This co-occurrence reflected clinical antibiotic resistance, and that the ARB which were isolated from hospital environments can be disseminated into the communities to cause health problems. ${ }^{5}$ It could increase the threat to community health because infection caused by ARB contributes to morbidity, mortality, and an increase in medical expenses; as many antibiotics are no longer effective. ${ }^{6}$ In recent years, emergences of ARB contamination in different hospital areas have been reported across the world. The gloves and gowns of healthcare personnel have been found to be contaminated with methicillin-resistant Staphylococcus aureus (MRSA) in intensive care units of 4 hospitals in United States of America $(\mathrm{USA})^{7}$; extended-spectrum $\beta$-lactamase (ESBL)-producing and carbapenemase-producing Enterobacteriaceae were both found in the bathroom sinks of a university hospital in France. ${ }^{8}$ In Thailand, HAls are common and are mainly caused by $A R B .^{3}$ Furthermore, research on ARB have mostly been conducted on clinical samples obtained from hospitalized patients. Clinical MRSA isolates concomitant with multidrug-resistant (MDR) phenotypes were found at a hospital in Bangkok' ampicillin and fluoroquinolones- resistant Vibrio parahaemolyticus isolates were obtained from diarrheal patients of a hospital in southern Thailand..$^{10}$ However, limited data exist on the contamination of bacteria as well as ARB in hospital environments. Grampositive cocci were mainly observed as airborne bacteria contaminating a large hospital center in southern Thailand. ${ }^{11}$ A high prevalence rate $(70.0 \%)$ of methicillin-resistant coagulase negative staphylococci (MR-CoNS) producing biofilms were found in a number of areas in a university hospital in Phitsanulok ${ }^{12}$; contamination of ESBL-producing Escherichia coli and/or Klebsiella pneumoniae in wastewater samples of community, general, and regional hospitals was also reported, even after treatment. ${ }^{13}$ Profiles of contaminated bacteria isolated from 10 different sampling points of inanimate surfaces and equipment of 12 subdistrict health-promoting hospitals (HPHs) in six subdistricts of Chiang Rai have also been revealed in one of our previous studies. ${ }^{14}$ In addition, that study was the first to report inspection results indicating bacterial contamination in sub-district level hospitals in Thailand as well as to provide primary data to support the further study of ARB occurrences in sub-district HPH environments, which is yet to be documented. To this end, nearly half of bacterial isolates (44.9\%; $153 / 341$ isolates) from our prior study were examined as the prime concern, regarding bacterial agents usually involved in HAls. ${ }^{15-17}$ The antibiotic susceptibility patterns (antibiogram profiles) of these bacteria including Staphylococcus spp., Streptococcus spp., members of the family Enterobacteriaceae, and Pseudomonas spp. were determined in this study. We anticipate these antibiogram profiles to be vital for extending trends of ARB from subdistrict level hospitals in both Chiang Rai, and Thailand.

\section{Material and Methods}

The Ethics Committees of the Chiang Rai Provincial Health Office, Thailand Ministry of Public Health approved the study protocol and all of its procedures and exempted 
the study from formal ethics review (CRPPHO No. 75/2563). This research does not contain any studies involving human or animal subjects. In addition, formal permission was obtained from the concerned authorities during sample collection.

The samples were collected from inanimate surfaces and equipment in 12 sub-district HPHs in six districts of Chiang Rai, Thailand, from January to March 2018. Ten different sampling points were selected for each sub-district $\mathrm{HPH}$. These 10 sampling points were chosen based on rates of exposure and due to being the most representative points of sub-district HPH services for clients and patients. The sampling points included: (1) bed and bed rails, (2) handwashing sinks, (3) medical devices/equipment, (4) portable treatment table and medical tray, (5) tables and chairs used by hospital personnel, (6) computers and keyboards used by hospital personnel, (7) toilet bowl dispensers, (8) toilet seat/toilet bowl areas, (9) toilet sinks and taps, and (10) knobs or latches of toilet doors. All sampling locations were found within 10-40 km of the laboratory (Medical Science Laboratory, Scientific and Technological Instrument Center (STIC), Mae Fah Luang University (MFU), Mueang District, Chiang Rai) for convenience. Collected samples were transferred to the laboratory within 2-3 hours (h).

Samples were collected using a sterile technique, by the swabbing method. ${ }^{18}$ The collected swab samples were immersed for propagation in a tryptic soy broth and incubated for $24 \mathrm{~h}$ at $37^{\circ} \mathrm{C}$. After incubation, these broth cultures were streaked onto differential and selective media: mannitol salt agar, blood agar, and MacConkey agar for the initial screening of suspected staphylococci, streptococci, and gram-negative bacteria (members of the family Enterobacteriaceae and Pseudomonas sp.), respectively. The inoculated plates were incubated at 37 ${ }^{\circ} \mathrm{C}$ for $24 \mathrm{~h}$ under aerobic conditions. The growth colonies were identified by Gram staining and standard biochemistry tests based on their biochemical properties. Briefly, Gram- positive isolates (Staphylococcus spp., Streptococcus spp.) were identified by catalase, and coagulase tests. Susceptibility to novobiocin (OXOID Ltd., Cheshire, United Kingdom) verified to differentiate $S$. epidermidis from $S$. saprophyticus. Members of Streptococcus spp. were examined by tests assessing growth in bile esculin medium, catalase and hemolytic reactions. The Optochin (OXOID Ltd.) test was performed to employ differentiation of viridans streptococci and $S$. pneumoniae. Members of the family Enterobacteriaceae and Pseudomonas spp. were identified by an oxidase test (Sigma-Aldrich, Missouri, USA), sugar fermentation (lactose and triple sugar ion (TSI); HiMedia Laboratories Pvt. Ltd., Mumbai, India), motile test, indole test, citrate test, urea test (HiMedia Laboratories Pvt. Ltd.), positive OF test, and pigment production on Pseudo F agar and Pseudo P agar (HiMedia Laboratories Pvt. Ltd.). ${ }^{19}$ Regular quality control of sterilization was performed according to the requirements of the Medical Science Laboratory, STIC of MFU. All isolated bacterial strains were preserved in a nutrient broth containing $20.0 \%(\mathrm{~V} / \mathrm{V})$ glycerol and were stored at freezing temperature for further analysis.

Antibiotic susceptibility testing was performed on Mueller-Hinton agar which based on the disk diffusion method ${ }^{20}$ according to the Clinical and Laboratory Standards Institute (CLSI, 2016) guidelines. ${ }^{21}$ The tested antibiotics discs (obtained from OXOID Ltd.) included cefoxitin (FOX; $30 \mu \mathrm{g})$, erythromycin $(\mathrm{E} ; 15 \mu \mathrm{g})$, clindamycin (DA; $2 \mu \mathrm{g})$, gentamycin $(\mathrm{CN} ; 10 \mu \mathrm{g})$, trimethroprim-sulfamethoxazole (SXT; 1.25/23.75 $\mu \mathrm{g})$, cefotaxime (CTX; $30 \mu \mathrm{g})$, ceftriaxone (CRO; $30 \mu \mathrm{g}$ ), vancomycin (VA; $30 \mu \mathrm{g}$ ), ampicillin (AMP; 10 $\mu \mathrm{g}$ ), ampicillin-clavulanic acid (AMC; 20/10 $\mu \mathrm{g}$ ), ampicillinsulbactam (SAM; 10/10 $\mu \mathrm{g}$ ), ciprofloxacin (CIP; $5 \mu \mathrm{g}$ ), amikacin (AK; $30 \mu \mathrm{g}$ ), and ceftazidime (CAZ; $30 \mu \mathrm{g})$. The susceptibility pattern to antibiotics (antibiogram profiles) was determined by measuring the diameter of the inhibition zone and comparing with the standards. The zone diameter 
interpretive standard recommended by the CLSI (2016) was presented as susceptible (S), intermediate (I), or resistant (R) of CLSI standard stains. ${ }^{21}$

\section{Results}

\section{Isolation rates}

A total of 153 isolates were examined for their relevant antibiotic resistance profiles. They were found to be contaminated at all sampling points (100.0\%) (Table 1). The most frequently contaminated points were the toilet seat/toilet bowl areas (16.3\%), followed by toilet sinks and taps (12.4\%), and the computers and keyboards of hospital personnel (11.1\%). The majority of these samples were gram-positive (85.0\%), with a predominance of CoAg-Neg staphylococci (S. epidermidis and S. saprophyticus, $66.0 \%$ and $13.1 \%$, respectively), followed by $S$. aureus $(3.3 \%)$ and viridans streptococci, a member of the Streptococcus species (2.6\%). Gram-negative bacteria primarily belonged to members of the family Enterobacteriaceae (E. coli, 2.0\%; K. pneumoniae, $0.7 \%$; others (not specified), $11.8 \%$ ), and $P$. aeruginosa $(0.7 \%)$. The estimated rates of contamination among the 10 sampling points of the sub-district HPHs varied from $10.0 \%$ to $100.0 \%$, with an average rate of $45.0 \%$.

\section{Susceptibility patterns of isolates to individual antibiotics}

The susceptibility of isolated Staphylococcus spp., Viridans streptococci, Enterobacteriaceae, and Pseudomonas spp. to antibiotics was tested against 5, 4, 6, and 3 primary categories of selected antibiotic discs, respectively. The results showed that the majority of the investigated isolates (ranging from $50.0 \%$ to $100.0 \%$ ), especially $P$. aeruginosa, were sensitive to several of the antibiotics tested (Table 2). However, CoAg-Neg staphylococci were found to be most resistant to erythromycin (35.5\%), followed by clindamycin
(26.5\%) and oxacillin (oxacillin-resistant-CoAg-Neg staphylococci; MRCoNS; 19.8\%). S. aureus isolates were the most resistant to clindamycin, whereas none of them was resistant to oxacillin. Viridans streptococci showed remarkable resistance to cefotaxime, ceftriaxone, and clindamycin $(75.0 \%$ each). E. coli was most resistant to ampicillin-sulbactam and ampicillin (33.3\% each). Similarly, one isolate of $K$. pneumoniae showed resistance to both ampicillin-sulbactam and ampicillin. Other members of Enterobacteriaceae (not specified, but excluding E. coli and $K$. pneumoniae) showed $44.4 \%$ (each) resistance to ampicillin and cefoxitin, and $38.9 \%$ to ampicillin-sulbactam but a low resistance to ciprofloxacin, trimethoprimsulfamethoxazole, and gentamycin. Fortunately, none of the isolated Enterobacteriaceae were ESBL-producing strains (data not shown).

\section{Antibiotic resistance phenotypes}

The antibiotic resistance patterns of the investigated bacteria showed that more than half $(60.0 \%)$ of $S$. aureus and $38.8 \%$ of CoAg-Neg staphylococci were sensitive to all of the antibiotics tested. Approximately $8.3 \%$ of CoAg-Neg staphylococci showed intermediate susceptibility, mostly to clindamycin and erythromycin. Two patterns of resistance were noted for $S$. aureus isolates, which were resistant to two and four of the drugs tested, respectively (20.0\% each), and all isolates of $S$. aureus were not oxacillin-resistant $S$. aureus (MRSA). CoAg-Neg staphylococci appeared to show six different resistance patterns, including resistance to a single drug (19.1\%; remarkable resistance to erythromycin $(9.9 \%)$, clindamycin $(5.0 \%)$, oxacillin and gentamycin $(1.7 \%$ each)), double drugs (17.4\%; remarkable resistance to clindamycin and erythromycin (8.3\%)), three drugs (4.8\%), four drugs (3.2\%), five drugs (5.0\%), and all drugs tested (3.3\%) (Table 3). 


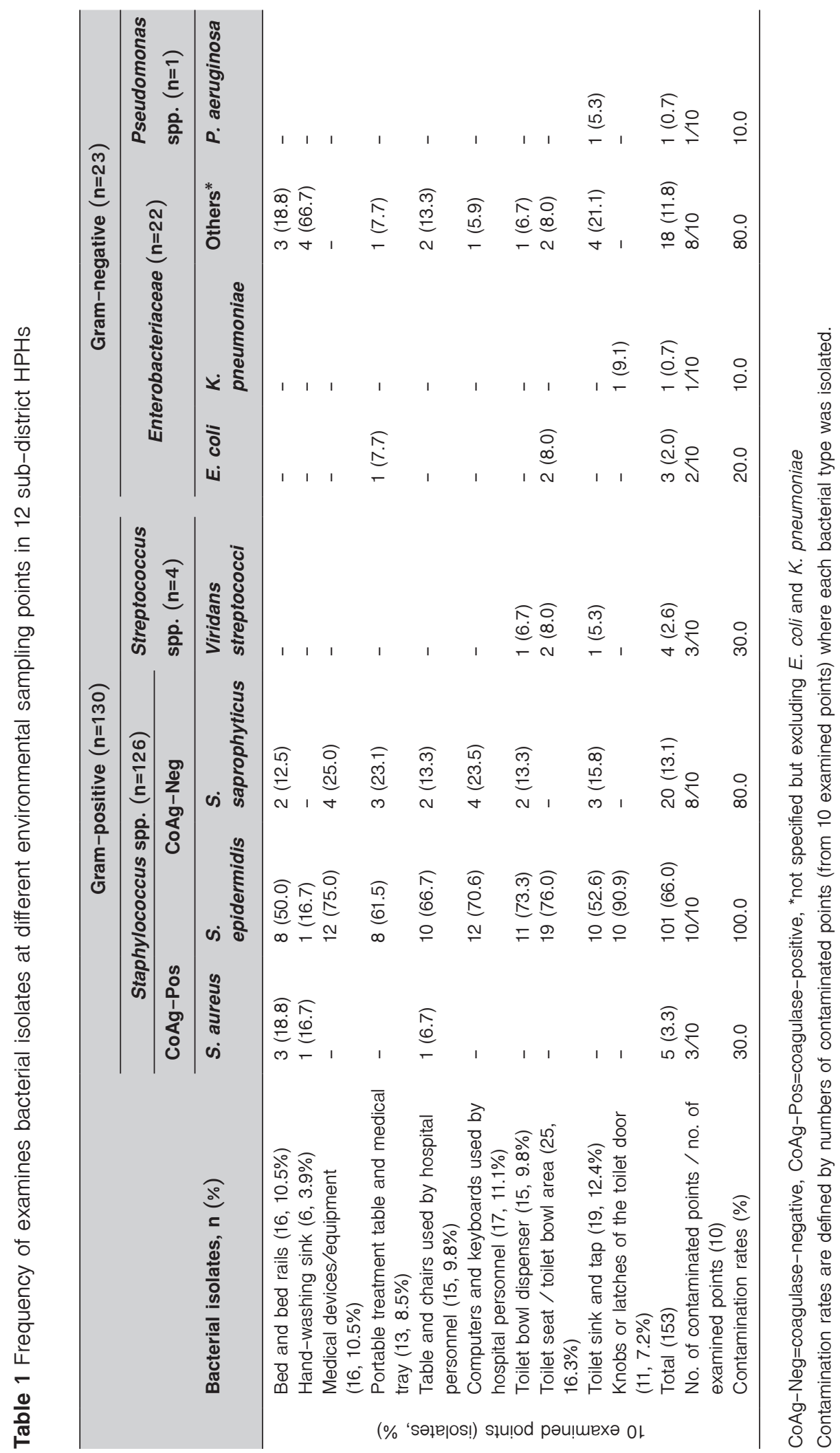


Table 2 Antibiotic susceptibility characteristics of examined bacteria

\begin{tabular}{|c|c|c|c|}
\hline \multirow{2}{*}{ Bacteria and antibiotic ( $\mu \mathrm{g} / \mathrm{disk})$} & \multicolumn{3}{|c|}{ Susceptibility level } \\
\hline & Sensitive & Intermediate & Resistant \\
\hline \multicolumn{4}{|l|}{ Staphylococcus spp. ( $\mathrm{N}=126)$} \\
\hline \multicolumn{4}{|l|}{ CoAg-Neg $(n=121)$} \\
\hline SXT; Trimethroprim-Sulfamethoxazone (1.25/23.75) & $79.3(96 / 121)$ & $1.7(2 / 121)$ & $19.0(23 / 121)$ \\
\hline FOX; Cefoxitin (30) & $88.4(107 / 121)$ & $0.0(0 / 121)$ & $11.6(14 / 121)$ \\
\hline CN; Gentamycin (10) & $85.1(103 / 121)$ & $0.8(1 / 121)$ & $14.1(17 / 121)$ \\
\hline DA; Clindamycin (2) & $66.1(80 / 121)$ & $7.4(9 / 121)$ & $26.5(32 / 121)$ \\
\hline E; Erythromycin (15) & $59.5(72 / 121)$ & $5.0(6 / 121)$ & $35.5(43 / 121)$ \\
\hline Oxacillin ${ }^{a, b}$ & $80.2(97 / 121)$ & $0.0(0 / 121)$ & $19.8(24 / 121)$ \\
\hline \multicolumn{4}{|l|}{ CoAg-Pos; Staphylococcus aureus $(n=5)$} \\
\hline SXT; Trimethroprim-Sulfamethoxazone (1.25/23.75) & $80.0(4 / 5)$ & $0.0(0 / 5)$ & $20.0(1 / 5)$ \\
\hline FOX; Cefoxitin (30) & $80.0(4 / 5)$ & $0.0(0 / 5)$ & $20.0(1 / 5)$ \\
\hline CN; Gentamycin (10) & $80.0(4 / 5)$ & $0.0(0 / 5)$ & $20.0(1 / 5)$ \\
\hline DA; Clindamycin (2) & $60.0(3 / 5)$ & $0.0(0 / 5)$ & $40.0(2 / 5)$ \\
\hline E; Erythromycin (15) & $80.0(4 / 5)$ & $0.0(0 / 5)$ & $20.0(1 / 5)$ \\
\hline Oxacillin ${ }^{a, b}$ & $0.0(0 / 5)$ & $0.0(0 / 5)$ & $0.0(0 / 5)$ \\
\hline \multicolumn{4}{|l|}{ Viridans streptococci $(\mathrm{N}=4)$} \\
\hline CTX; Cefotaxime (30) & $25.0(1 / 4)$ & $0.0(0 / 4)$ & $75.0(3 / 4)$ \\
\hline CRO; Ceftriaxone (30) & $25.0(1 / 4)$ & $0.0(0 / 4)$ & $75.0(3 / 4)$ \\
\hline E; Erythromycin (15) & $75.0(3 / 4)$ & $0.0(0 / 4)$ & $25.0(1 / 4)$ \\
\hline DA; Clindamycin (2) & $25.0(1 / 4)$ & $0.0(0 / 4)$ & $75.0(3 / 4)$ \\
\hline VA; Vancomycin (30) & $75.0(3 / 4)$ & $0.0(0 / 4)$ & $25.0(1 / 4)$ \\
\hline \multicolumn{4}{|l|}{ Enterobacteriaceae $(\mathrm{N}=22)$} \\
\hline \multicolumn{4}{|l|}{ Escherichia coli $(\mathrm{n}=3)$} \\
\hline FOX; Cefoxitin (30) & $100.0(3 / 3)$ & $0.0(0 / 3)$ & $0.0(0 / 3)$ \\
\hline CIP; Ciprofloxacin (5) & $100.0(3 / 3)$ & $0.0(0 / 3)$ & $0.0(0 / 3)$ \\
\hline SXT; Trimethroprim-Sulfamethoxazone (1.25/23.75) & $100.0(3 / 3)$ & $0.0(0 / 3)$ & $0.0(0 / 3)$ \\
\hline CN; Gentamycin (10) & $100.0(3 / 3)$ & $0.0(0 / 3)$ & $0.0(0 / 3)$ \\
\hline SAM; Ampicillin-Sulbactam (10/10) & $66.7(2 / 3)$ & $0.0(0 / 3)$ & $33.3(1 / 3)$ \\
\hline AMP; Ampicillin (10) & $66.7(2 / 3)$ & $0.0(0 / 3)$ & $33.3(1 / 3)$ \\
\hline AMC; Ampicillin-Clavulanic acid (20/10) & $100.0(3 / 3)$ & $0.0(0 / 3)$ & $0.0(0 / 3)$ \\
\hline \multicolumn{4}{|l|}{ Klebsiella pneumoniae $(\mathrm{n}=1)$} \\
\hline FOX; Cefoxitin (30) & $100.0(1 / 1)$ & $0.0(0 / 1)$ & $0.0(0 / 1)$ \\
\hline CIP; Ciprofloxacin (5) & $100.0(1 / 1)$ & $0.0(0 / 1)$ & $0.0(0 / 1)$ \\
\hline SXT; Trimethroprim-Sulfamethoxazone (1.25/23.75) & $100.0(1 / 1)$ & $0.0(0 / 1)$ & $0.0(0 / 1)$ \\
\hline CN; Gentamycin (10) & $100.0(1 / 1)$ & $0.0(0 / 1)$ & $0.0(0 / 1)$ \\
\hline SAM; Ampicillin-Sulbactam (10/10) & $0.0(0 / 1)$ & $0.0(0 / 1)$ & $100.0(1 / 1)$ \\
\hline AMP; Ampicillin (10) & $0.0(0 / 1)$ & $0.0(0 / 1)$ & $100.0(1 / 1)$ \\
\hline AMC; Ampicillin-Clavulanic acid (20/10) & $100.0(1 / 1)$ & $0.0(0 / 1)$ & $0.0(0 / 1)$ \\
\hline \multicolumn{4}{|l|}{ Others; not specified $(n=18)$} \\
\hline FOX; Cefoxitin (30) & $50.0(9 / 18)$ & $5.6(1 / 18)$ & $44.4(8 / 18)$ \\
\hline CIP; Ciprofloxacin (5) & $94.4(17 / 18)$ & $0.0(0 / 18)$ & $5.6(1 / 18)$ \\
\hline SXT; Trimethroprim-Sulfamethoxazone (1.25/23.75) & $94.4(17 / 18)$ & $0.0(0 / 18)$ & $5.6(1 / 18)$ \\
\hline CN; Gentamycin (10) & $94.4(17 / 18)$ & $0.0(0 / 18)$ & $5.6(1 / 18)$ \\
\hline SAM; Ampicillin-Sulbactam (10/10) & $61.1(11 / 18)$ & $0.0(0 / 18)$ & $38.9(7 / 18)$ \\
\hline AMP; Ampicillin (10) & $50.0(9 / 18)$ & $5.6(1 / 18)$ & $44.4(8 / 18)$ \\
\hline AMC; Ampicillin-Clavulanic acid (20/10) & $66.7(12 / 18)$ & $0.0(0 / 18)$ & $33.3(6 / 18)$ \\
\hline \multicolumn{4}{|l|}{ Pseudomonas spp. $(\mathrm{N}=1)$} \\
\hline \multicolumn{4}{|l|}{ Pseudomonas aeruginosa } \\
\hline CIP; Ciprofloxacin (5) & $100.0(1 / 1)$ & $0.0(0 / 1)$ & $0.0(0 / 1)$ \\
\hline AK; Amikacin (30) & $100.0(1 / 1)$ & $0.0(0 / 1)$ & $0.0(0 / 1)$ \\
\hline CAZ; Ceftazidime (30) & $100.0(1 / 1)$ & $0.0(0 / 1)$ & $0.0(0 / 1)$ \\
\hline CN; Gentamycin (10) & $100.0(1 / 1)$ & $0.0(0 / 1)$ & $0.0(0 / 1)$ \\
\hline
\end{tabular}

Values are \% (n/N), CoAg-Neg=coagulase-negative; CoAg-Pos=coagulase-positive

${ }^{a}$ Oxacillin disk testing is not reliable; $30 \mu \mathrm{g}$ cefoxitin as a surrogate agent for oxacillin; report oxacillin susceptible or resistant based on the cefoxitin result; ${ }^{b}$ Oxacillin-resistant is considered as methicillin-resistant, CLSI (2016) ${ }^{21}$ 
Table 3 Antibiogram profiles of Staphylococcus spp. ( $\mathrm{N}=126)$

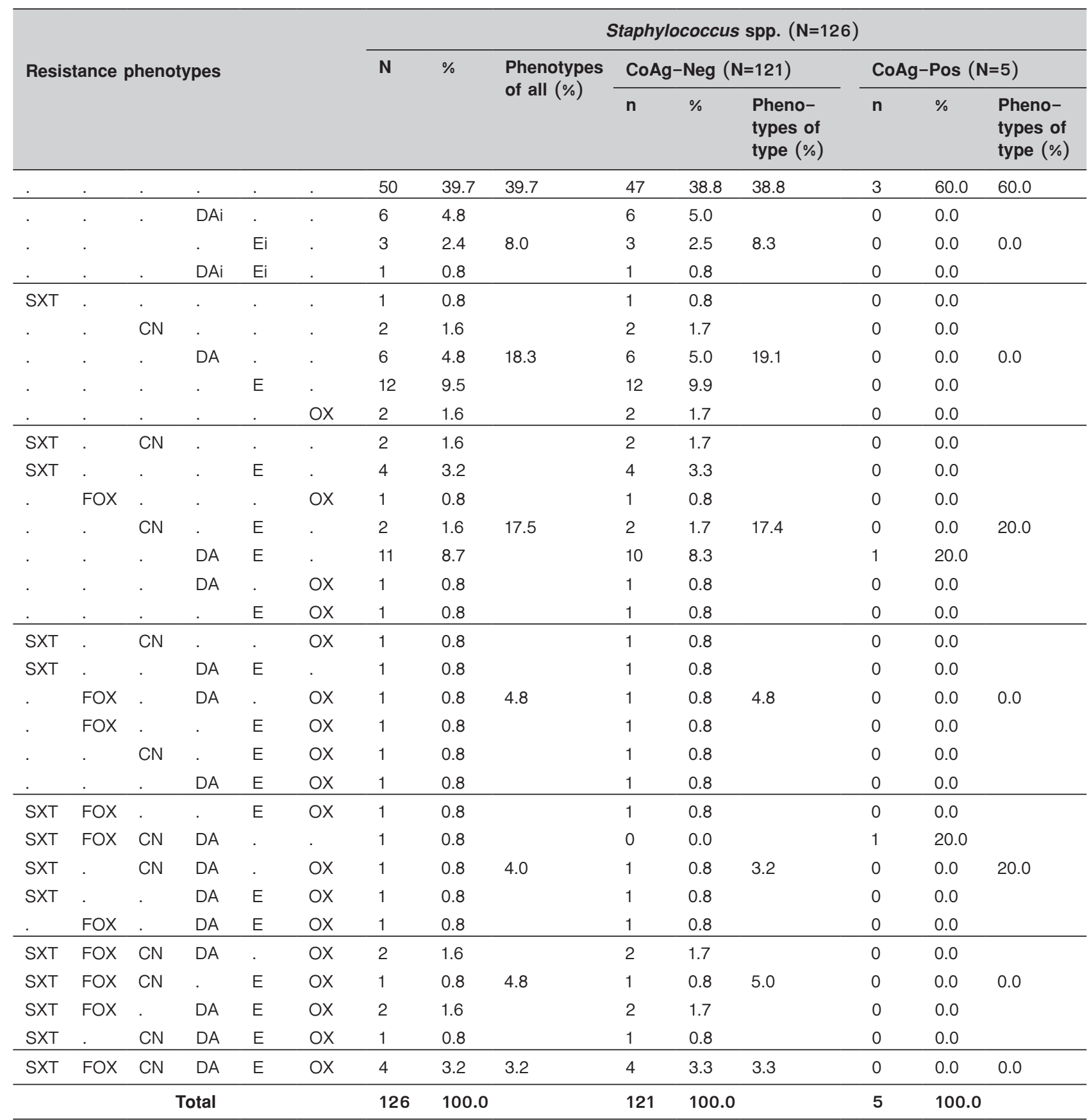

=sensitive, SXT=Trimethroprim-Sulfamethoxazone-resistant, FOX=Cefoxitin-resistant, CN=Gentamycin-resistant, DA=Clindamycin-resistant, $\mathrm{DA}=$ Clindamycin-intermediate, $\mathrm{E}=$ Erythromycin-resistant, Ei=Erythromycin-intermediate, OX=Oxacillin-resistant [Oxacillin disk testing is not reliable; $30 \mu \mathrm{g}$ cefoxitin as a surrogate agent for oxacillin; report oxacillin susceptible or resistant based on the cefoxitin result and oxacillinresistant is considered as methicillin-resistant, CLSI $(2016)^{21}$ ] 
The phenotypes of resistance for isolated members of the family Enterobacteriaceae showed that $E$. coli and K. pneumoniae exhibited a similar pattern of resistance to double drugs tested with ampicillin-sulbactam and ampicillin (33.3\% and 100.0\%, respectively). Others (not specified, except E. coli and K. pneumoniae) displayed five patterns of resistance to a single drug (27.9\%; remarkable resistance to ampicillin $(16.7 \%)$ ), double drugs $(11.2 \%)$, three drugs (16.7\%), four drugs (11.1\%), and all six drugs tested (5.6\%) (Table 4).

Viridans streptococci showed notable resistance to two and three drugs, which covered all categories tested. With the exception of other isolated bacteria, only one isolate of $P$. aeruginosa showed distinctive susceptibility to all the antibiotics tested (Table 5).

\section{Categorization of isolates based on their anti-}

\section{biotic resistance profiles}

Among 153 bacterial isolates, 85 (55.6\%) were resistant isolates categorized by three profiles: single drug resistant (SDR; 18.3\%), double drug resistant (DDR; 18.3\%), and multi-drug resistant (defined as resistant to three or more antimicrobial classes ${ }^{22}$, MDR; 19.0\%) bacteria (Table 6). The $P$. aeruginosa isolate was susceptible to all the antibiotics tested. MDR phenotypes were most common in CoAg-Neg staphylococci (13.1\%), followed by other Enterobacteriaceae (not specified, except E. coli and $K$. pneumoniae) (3.9\%), Viridans streptococci (1.3\%) and S. aureus $(0.7 \%)$. The distribution of ARB from different sampling points is shown in Figure 1. ARBs were found at all sampling points (100.0\%), especially the toilet seat/ toilet bowl area (17.6\%), followed by the toilet sink and tap (14.1\%), and the toilet bowl dispenser (12.9\%). MDR isolates were abundant in all sampling points, except the knobs or latches of the toilet doors. Among each sampling points, the highest proportion (60.0\%) of MDR isolates were obtained from the bed and bed rails, and hand-washing sink (equally), followed by the computers and keyboards used by hospital personnel $(50.0 \%)$.

\section{Discussion}

In this study, the bacterial agents usually involved in HAls, namely Staphylococcus spp., Streptococcus spp. (viridans streptococci), members of the family Enterobacteriaceae (E. coli, K. pneumoniae, and others), and Pseudomonas spp. contaminations on surface areas and inanimate equipment in the 12 sub-district HPHs in Chiang Rai were examined for their antibiotic resistance profiles. CoAg-Neg staphylococci (particularly S. epidermidis) were the most frequently isolated, consistent with a previous study. ${ }^{23}$ This was not unexpected because they are mainly normal skin flora and especially $S$. epidermidis has a high potential to form biofilms on surfaces. ${ }^{24}$ In addition, Kramer et al. $^{25}$ found that most gram-positive bacteria, such as staphylococci and the strains of streptococci, many gram-negative bacteria, notably E. coli, Klebsiella spp., and $P$. aeruginosa survived for months on dry inanimate surfaces. Although many of these bacteria can be disregarded as harmless commensals, the average contamination rate of $45.0 \%$ in the present study was quite high. This could be attributed to bacterial shedding leading into their transmittion on inanimate equipment and surface areas in sub-district HPHs, which then become HAls. To the best of our knowledge, HAls are common in Thailand and most are caused by ARB. ${ }^{3}$ Subsequently, the antibiotic susceptibility patterns of the isolated bacteria in the present study were determined.

Over half $(61.2 \%)$ of CoAg-Neg staphylococci isolates in this study developed resistance to five categories of antibiotics (folate pathway inhibitors, anti-staphylococcal $\beta$-lactams, aminoglycosides, lincosamides, and macrolides) (Table 3), notably high resistance to macrolides (erythromycin, 9.9\%) and lincosamide (clindamycin, 5.0\%), which have been widely used in the treatment of staphylococcal infections. 


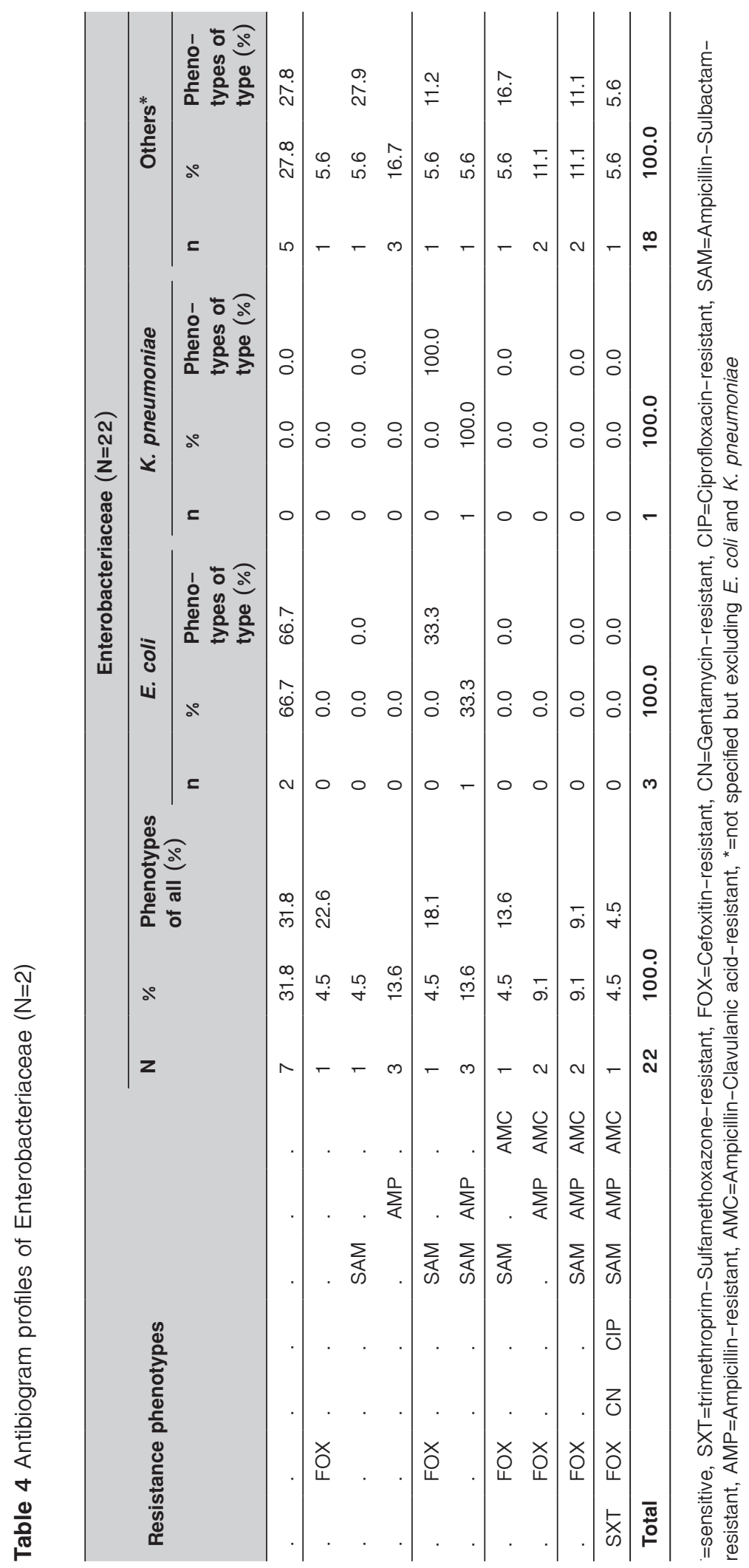


Table 5 Antibiogram profiles of Viridans streptococci and Pseudomonas aerugino

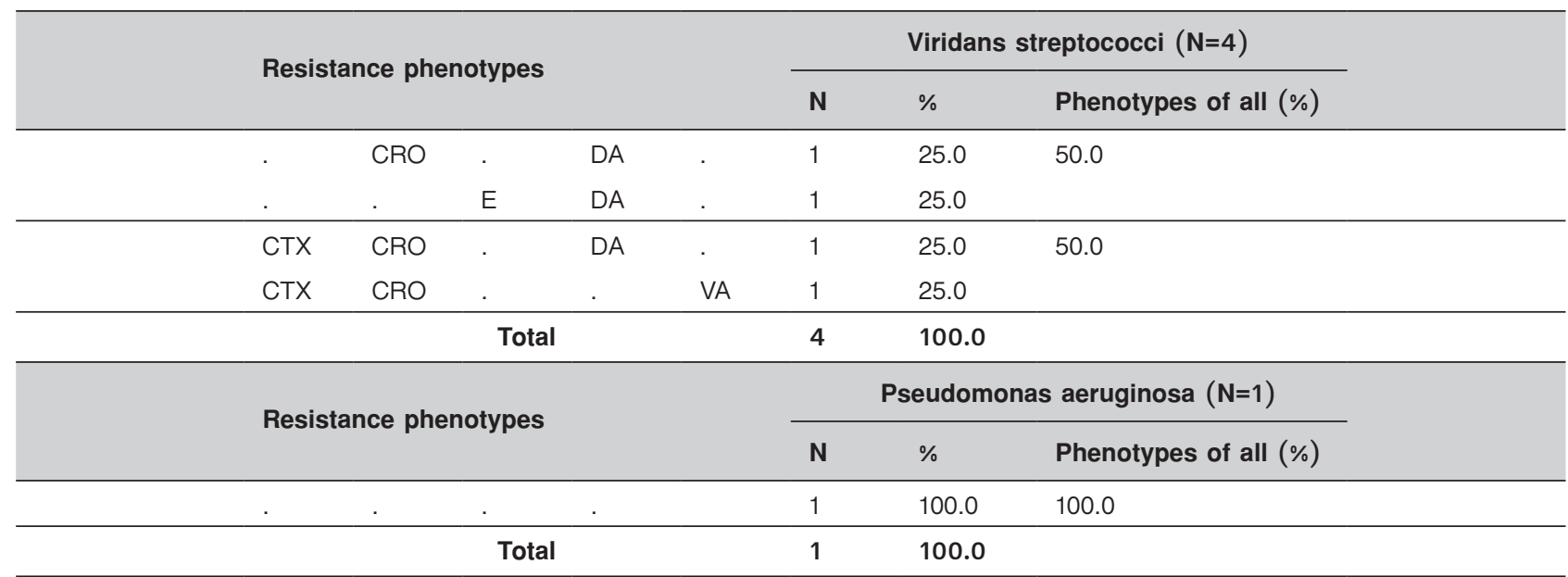

=sensitive, Antibiotic testing for Viridans streptococci: CTX=cefotaxime-resistant, CRO=ceftriaxone-resistant, E=erythromycin-resistant, $\mathrm{DA}=$ clindamycin-resistant, $\mathrm{VA}=$ vancomycin-resistant

Antibiotic testing for Pseudomonas aeruginosa: $\mathrm{CIP}=$ ciprofloxacin-resistant, $\mathrm{AK}=$ amikacin-resistant, $\mathrm{CAZ}=$ ceftazidime-resistant, $\mathrm{CN}=$ gentamycin-resistant

Table 6 Frequency distribution of antibiogram profiles of examined bacteria

\begin{tabular}{|c|c|c|c|c|c|c|}
\hline \multirow{2}{*}{ Isolated organisms } & \multicolumn{2}{|c|}{ SDR } & \multicolumn{2}{|c|}{ DDR } & \multicolumn{2}{|c|}{ MDR } \\
\hline & $\%$ of type & $\%$ of all & $\%$ of type & $\%$ of all & $\%$ of type & $\%$ of all \\
\hline S. aureus (5) & 0 & 0 & $1 / 5(20.0)$ & $1 / 153(0.7)$ & $1 / 5(20.0)$ & $1 / 153(0.7)$ \\
\hline CoAg-Neg staphylococci (121) & 23/121 (19.0) & 23/153 (15.0) & $21 / 121(17.4)$ & 21/153 (13.7) & $20 / 121(16.5)$ & $20 / 153(13.1)$ \\
\hline Viridans streptococci (4) & 0 & 0 & $2 / 4(50.0)$ & $2 / 153(1.3)$ & $2 / 4(50.0)$ & $2 / 153(1.3)$ \\
\hline Escherichia coli (3) & 0 & 0 & $1 / 3(33.3)$ & $1 / 153(0.7)$ & 0 & 0 \\
\hline Klebsiella pneumoniae (1) & 0 & 0 & $1 / 1(100.0)$ & $1 / 153(0.7)$ & 0 & 0 \\
\hline Others Enterobacteriaceae* (18) & $5 / 18(27.8)$ & 5/153 (3.3) & $2 / 18(11.1)$ & $2 / 153(1.3)$ & 6/18 (33.3) & 6/153 (3.9) \\
\hline Pseudomonas aeruginosa (1) & 0 & 0 & 0 & 0 & 0 & 0 \\
\hline Total (153) & & $\begin{array}{l}28 / 153 \\
(18.3)\end{array}$ & & $\begin{array}{l}28 / 153 \\
(18.3)\end{array}$ & & $\begin{array}{l}29 / 153 \\
(19.0)\end{array}$ \\
\hline
\end{tabular}

Values are $\%(\mathrm{n} / \mathrm{N})$ for SDR, DDR, and MDR, CoAg-Neg=coagulase-negative, ${ }^{*}=$ not specified but excluding $E$. coli and $K$. pneumoniae, $\mathrm{SDR}=$ single drug resistant, $\mathrm{DDR}=$ double drugs resistant, $\mathrm{MDR}=$ multi-drugs resistant (MDR, resistant to three or more antimicrobial classes ${ }^{22}$ ) 


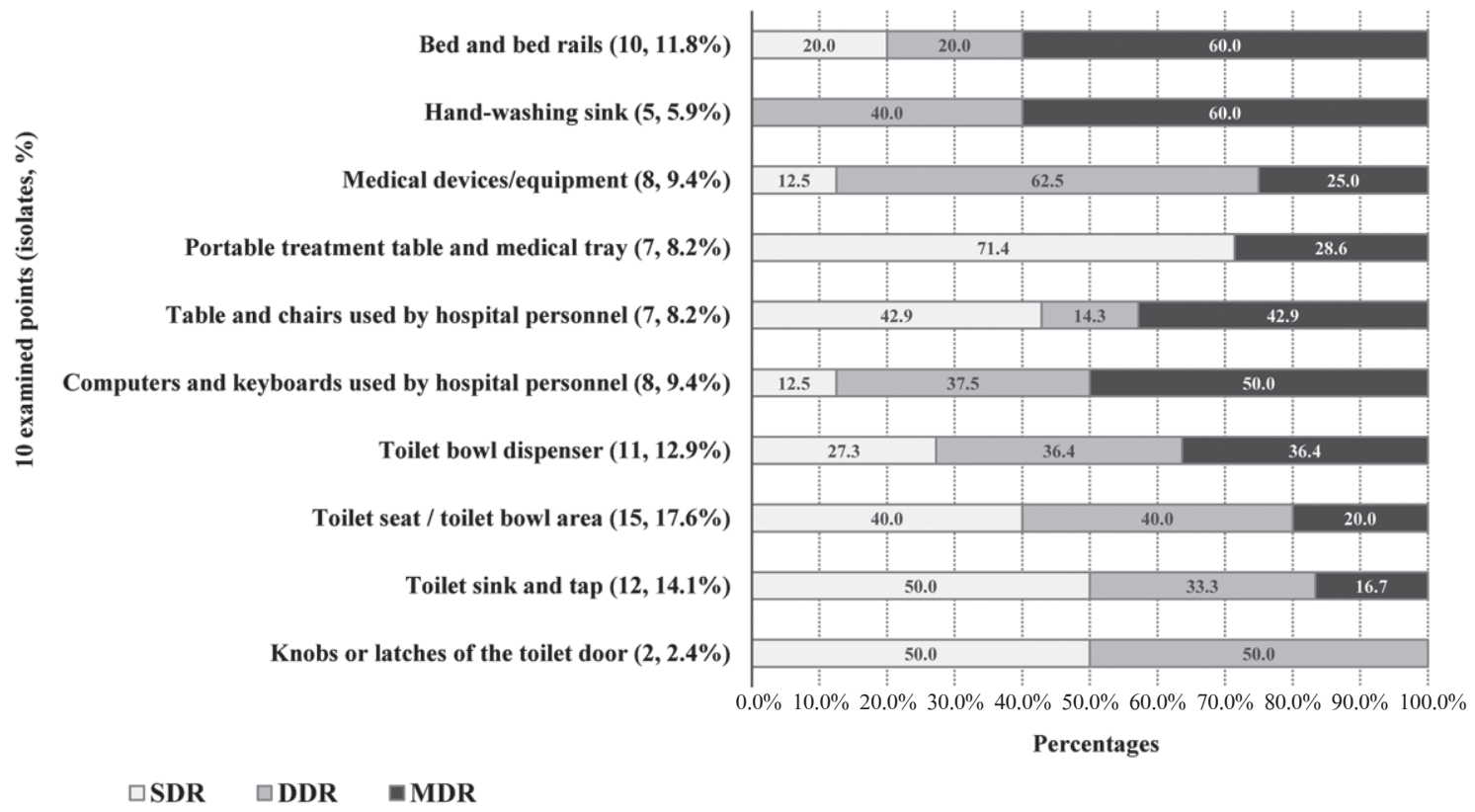

Total ARB isolates=85/153 (55.6\%); SDR=single drug resistant, DDR=double drugs resistant, MDR=multi-drugs resistant (MDR=resistant to three or more antimicrobial classes ${ }^{22}$ )

Figure 1 Distribution of ARB found at different environmental sampling points in 12 sub-district HPHs

This was associated with their DDR profiles, which were remarkable for clindamycin and erythromycin combinations (8.3\%). Similarly, $20.0 \%$ of the $S$. aureus isolates also showed high resistance to these two drugs. This could be because CoAg-Neg staphylococci are potential sources of antibiotic resistance genes, which are capable of transferring these genes to $S$. aureus. ${ }^{26}$ Moreover, a previous study demonstrated that macrolide resistance was more prevalent in CoAg-Neg staphylococci. ${ }^{27}$ Erythromycin and clindamycin are antibiotics that inhibit protein synthesis by binding to the $50 \mathrm{~S}$ ribosomal subunits of bacterial cells. Resistance to both drugs can occur through the methylation of the ribosomal target site. $^{28}$ In addition, macrolide resistance may be constitutive or inducible in the presence of either a macrolide or lincosamide inducer. ${ }^{29}$ The rate of antistaphylococcal $\beta$-lactams (oxacillin) resistance showed that MRCoNS was $19.8 \%$, whereas no MRSA was detected.
This may be because of the greater proportion of CoAgNeg staphylococci isolates than $S$. aureus isolates in the present study. In addition, these rates are lower than those of previous studies which reported $70.0 \%$ of $\mathrm{MRCoNS}^{12}$, and $50.0 \%$ of MRSA strains found in hospital environments. ${ }^{30}$

Viridans streptococci isolated from this study showed $100.0 \%$ (4/4) resistance to four categories of antibiotics: cephems, macrolides, lincosamides, and glycopeptides. High levels of resistance to individual antibiotics were observed against cefotaxime, ceftriaxone, and clindamycin (75.0\% each) (Table 2), which was higher than the rates of resistance to ceftazidime and ceftriaxone $(53.0 \%$ and $22.0 \%$, respectively), and the $18.0 \%$ resistance to clindamycin reported by another study. ${ }^{31}$ DDR and MDR profiles were noticeably aligned with a combination of these three distinct classes (ceftriaxone-clindamycin, cefotaxime-ceftriaxoneclindamycin, $25.0 \%$ each) (Table 5). In contrast, low 
resistance to erythromycin and vancomycin was observed. This was in agreement with the findings of a prior study ${ }^{32}$, which suggested the use of glycopeptide (vancomycinbased regimens) for the empirical antibiotic treatment of viridans streptococcal infection.

For gram-negative isolates, $67.9 \%$ of Enterobacteriaceae appeared to be resistant to six categories of antibiotics: cephamycins, fluoroquinolones, folate pathway inhibitors, aminoglycosides, penicillins (and penicillins + $\beta$-lactamase inhibitors (Table 4). E. coli and K. pneumoniae isolates were notable for their resistance to ampicillin and ampicillin-sulbactam (Table 2), which is in agreement with a previous study. ${ }^{33}$ Their DDR patterns were associated with these two antibiotic combinations (Table 4). In addition, different resistance profiles were observed among other members of Enterobacteriaceae (not specified, except $E$. coli and K. pneumoniae) because of the higher proportion and variation in strains, which was limited to identification in this study. They were most resistant to ampicillin (16.7\%), followed by cefoxitin and ampicillin-sulbactam (5.6\% each), in combination with their DDR and MDR profiles for these three different classes, together with ampicillin-clavulanic acid (Table 4). This might be because members of Enterobacteriaceae are highly resistant to commonly used $\beta$-lactam-based antibiotics (including ampicillin, ampicillinsulbactam, and ampicillin-clavulanic acid). ${ }^{34}$ Beyond limited information about the susceptibility profiles of common Enterobacteriaceae, it should be noted that ESBL production was not observed in any of the Enterobacteriaceae isolates in this study (data not shown). This may be because of the limited types of antibiotics used in sub-district HPHs, since their services focus generally on providing only primary care treatments for local community people, with no in-patient services.

Unlike other isolates in our study, $P$. aeruginosa was $100.0 \%$ sensitive to all categories of antibiotics tested. This was exceptional because $P$. aeruginosa is well known for its ability to be multidrug resistant, as supported by an earlier study. ${ }^{35}$ The small number of $P$. aeruginosa isolates in this study may have limited its resistance profile (only one isolate was detected in samples collected from 12 sub-district HPHs).

Nearly half of all isolates (85/153; 55.6\%) were resistant to the selected antibiotic categories (Table 6). These ARB were found distributed in all sampling points, mainly on the surfaces of toilet areas, whereas most of MDR isolates was abundant on surfaces and equipment located in the patient treatment areas of the nursing rooms (Figure 1). This could reflect that routine daily disinfection practices on particular surfaces and equipment might not be adequate. The overall rates of SDR, DDR, and MDR seem to be associated with the proportion of isolated types, indicating that CoAg-Neg staphylococci (13.1\%) were notably MDR phenotypes. Although the MDR rate of $19.0 \%$ reported in this study was much lower than the MDR rates reported by others $\left(77.0 \%{ }^{36}, 79.4 \%{ }^{30}\right.$, and $\left.81.5 \%{ }^{37}\right)$, the occurrence of MDR phenotypes should be taken into consideration. In regards to this study, "worrying" rates of antibiotic resistance in bacteria contaminating different environmental samples of the sub-district HPHs were observed. This could indicate that sub-district HPHs may serve as sites of HAls caused by bacteria which are capable of resistance to multiple categories of antibiotic drugs. Transmission of these ARB from sub-district HPHs to the community via contact between sub-district $\mathrm{HPH}$ personnel and their patients or clients, was inevitable. As a result, antibiotic resistance, especially MDR phenotypes, could become a problem in local communities since infections caused by these bacteria are more difficult or impossible to treat with currently available antibiotics, leading to greater morbidity and mortality, and resulting in higher healthcare costs. ${ }^{36}$ It is worth noting that antibiotic stewardship and other preventive strategies are recommended to prevent the ARB transmission. ${ }^{34}$ Practicing infection control standards to ensure the cleaning and use of cleaning agents on sub-district $\mathrm{HPH}$ environments, and keep checking on 
antibiotic use and its resistance might be essential. For sub-district HPHs personnel, maintaining personal hygiene especially hand decontamination is required with proper hand disinfectants before and after being in contact with their patients or clients. ${ }^{38}$ Furthermore, the surveillance of antibiotic susceptibility should be implemented in sub-district $\mathrm{HPHs}$, since these are necessary to provide healthcare services to the community. ${ }^{39}$

\section{Conclusion}

This study is the first to provide original data on the antibiogram profiles of the bacterial agents usually involved in HAls found on surface areas and inanimate equipment of sub-district level hospitals in Thailand. By collecting samples from 12 sub-district HPHs in Chiang Rai, we highlighted the occurrence of ARB, especially MDR phenotypes, and pointed out the high chance of ARB crosscontamination and cross-infection, which poses a threat to the health of individuals in the nearby community when no preventive measures to control or limit bacterial spread are implemented. This study provides a basis for further studies on antibiotic stewardship and the surveillance of antibiotic susceptibility in sub-district HPHs in order to allow the development of measures that support consequential community healthcare in the near future.

\section{Acknowledgement}

The authors are grateful to the directors of the 12 sub-district HPHs in Chiang Rai for their sampling support.

\section{Funding sources}

This work was supported by the Mae Fah Luang University research fund (fiscal year 2018).

\section{Conflict of interest}

None declared.

\section{References}

1. Suleyman G, Alangaden G, Bardossy AC. The role of environmental contamination in the transmission of nosocomial pathogens and healthcare-associated infections. Curr Infect Dis Rep 2018;20:1-11.

2. Facciolà A, Pellicanò GF, Visalli G, Paolucci IA, Venanzi RE, Ceccarelli $\mathrm{M}$, et al. The role of the hospital environment in the healthcare-associated infections: a general review of the literature. Eur Rev Med Pharmacol Sci 2019;23:1266-78.

3. Thamlikitkul V, Rattanaumpawan P, Boonyasiri A, Pumsuwan V, Judaeng $\mathrm{T}$, Tiengrim $\mathrm{S}$, et al. Thailand antimicrobial resistance containment and prevention program. J Glob Antimicrob Resist 2015;3:290-4.

4. Webb GF, D'Agata EM, Magal P, Ruan S. A model of antibioticresistant bacterial epidemics in hospitals. Proc Natl Acad Sci USA 2005;102:13343-8.

5. Cai L, Sun J, Yao F, Yuan Y, Zeng M, Zhang Q, et al. Antimicrobial resistance bacteria and genes detected in hospital sewage provide valuable information in predicting clinical antimicrobial resistance. Sci Total Environ 2021;15:148815.

6. Khamsarn S, Nampoonsak Y, Busamaro S, Tangkoskul T, Seenama C, Rattanaumpawan P, et al. Epidemiology of antibiotic use and antimicrobial resistance in selected communities in Thailand. J Med Assoc Thai 2016;99:270-5

7. O’Hara LM, Calfee DP, Miller LG, Pineles L, Magder LS, Johnson JK, et al. Optimizing contact precautions to curb the spread of antibiotic-resistant bacteria in hospitals: a multicenter cohort study to identify patient characteristics and healthcare personnel interactions associated with transmission of methicillin-resistant Staphylococcus aureus. Clin Infect Dis 2019; 69(Suppl 3):S171-7.

8. Sevin T, Goldstein V, Lolom I, Lenne F, Gaudonnet Y, Baptiste $\mathrm{AL}$, et al. Bathroom contamination by antibiotic-resistant Enterobacterales (ESBLPE and CPE): an experimental study. J Hosp Infect 2020;106:271-6.

9. Pipobwatthana P, Pummangura C, Jaroennon S, Tribuddharat C, Thuncharoon H, So-Ngern A, et al. Prevalence and antibiogram profiles of Staphylococcus aureus isolates from patients at Taksin Hospital, Bangkok, Thailand (January 2019May 2020). Southeast Asian J Trop Med Public Health 2021; 52:230-9. 
10. Preeprem S, Bhoopong P, Srinitiwarawong K, Vuddhakul V, Mittraparp-arthorn P. Antibiogram profiles and virulence characteristics of pandemic Vibrio parahaemolyticus isolates from diarrheal patients in Hat Yai hospital, Southern Thailand. Southeast Asian J Trop Med Public Health 2019;50:132-45.

11. Onmek N, Kongcharoen J, Singtong A, Penjumrus A, Junnoo S. Environmental factors and ventilation affect concentrations of microorganisms in hospital wards of Southern Thailand. J Environ Public Health 2020;2020. doi: 10.1155/2020/7292198.

12. Seng R, Kitti T, Thummeepak R, Kongthai $P$, Leungtongkam U, Wannalerdsakun S, Sitthisak S. Biofilm formation of methicillinresistant coagulase negative staphylococci (MR-CoNS) isolated from community and hospital environments. PLoS One 2017;12:e0184172.

13. Thamlikitkul V, Tiengrim S, Thamthaweechok N, Buranapakdee P, Chiemchaisri W. Contamination by antibiotic-resistant bacteria in selected environments in Thailand. Int $\mathrm{J}$ Environ Res Public Health 2019;16:3753.

14. Chansareewittaya K, Krajangcharoensakul S. Existence of bacterial contamination on inanimate surfaces and equipment in sub-district health-promoting hospitals in Chiang Rai, Thailand. J Curr Sci Technol 2021;11:355-66.

15. Ducel G, Fabry J, Nicolle L. Prevention of hospital-acquired infections: a practical guide. $2^{\text {nd }}$ ed. Geneva: World Health Organization; 2002.

16. Horan TC, Andrus M, Dudeck MA. CDCANSN surveillance definition of health care-associated infection and criteria for specific types of infections in the acute care setting. Am J Infect Control 2008;36:309-32.

17. Khan HA, Ahmad A, Mehboob R. Nosocomial infections and their control strategies. Asian Pac J Trop Biomed 2015;5:509-14.

18. Department of Medical Sciences, Ministry of Public Health, Thailand. Sampling and safety guide. Bangkok: Text and Journal; 2014.

19. Brown A, Smith H. Benson's microbiological applications: laboratory manual in general microbiology. $13^{\text {th }}$ ed. New York: The McGraw-Hill; 2012.

20. Bauer AW, Kerby WMM, Sherris JC, Turk M. Antibiotic susceptibility testing by a standardized single disk method. Am J Clin Pathol 1996;45:493-6.

21. CLSI. Performance Standards for Antimicrobial Susceptibility Testing. $26^{\text {th }}$ ed. CLSI supplement M100S. Wayne, PA: Clinical and Laboratory Standards Institute; 2016.
22. Magiorakos AP, Srinivasan A, Carey RB, Carmeli $Y$, Falagas ME, Giske CG, et al. Multidrug-resistant, extensively drugresistant and pandrug-resistant bacteria: an international expert proposal for interim standard definitions for acquired resistance. Clin Microbiol Infect 2012;18:268-81.

23. Lalami AE, Touijer H, Ettayebi M, Benchemsi N. Microbiological monitoring of environment surfaces in a hospital in Fez city, Morocco. J Mater Environ Sci 2016;7:123-30.

24. Shaheen A, Baqai R. Biofilm Formation by Environmental Microbes Isolated from Hospitals in Karachi, Pakistan. Am Sci Res J Eng Technol Sci 2016;15:240-51.

25. Kramer A, Schwebke I, Kampf G. How long do nosocomial pathogens persist on inanimate surfaces? A systematic review. BMC Infect Dis 2006;6:1-8.

26. Juda M, Chudzik-Rzad B, Malm A. The prevalence of genotypes that determine resistance to macrolides, lincosamides, and streptogramins B compared with spiramycin susceptibility among erythromycin-resistant Staphylococcus epidermidis. Mem Inst Oswaldo Cruz 2016;111:155-60.

27. Lina G, Quaglia A, Reverdy ME, Leclercq R, Vandenesch F, Etienne J. Distribution of genes encoding resistance to macrolides, lincosamides, and streptogramins among staphylococci. Antimicrob Agents Chemother 1999;43:1062-6.

28. Steward CD, Raney PM, Morrell AK, Williams PP, McDougal LK, Jevitt $L$, et al. Testing for induction of clindamycin resistance in erythromycin-resistant isolates of Staphylococcus aureus. J Clin Microbiol 2005;43:1716-21.

29. Patel M, Waites KB, Moser SA, Cloud GA, Hoesley CJ. Prevalence of inducible clindamycin resistance among community-and hospital-associated Staphylococcus aureus isolates. J Clin Microbiol 2006;44:2481-4.

30. Tajeddin E, Rashidan M, Razaghi M, Javadi SS, Sherafat SJ, Alebouyeh M, et al. The role of the intensive care unit environment and health-care workers in the transmission of bacteria associated with hospital acquired infections. $J$ Infect Public Health 2016;9:13-23.

31. Poeschl PW, Spusta L, Russmueller G, Seemann R, Hirschl A, Poeschl E, et al. Antibiotic susceptibility and resistance of the odontogenic microbiological spectrum and its clinical impact on severe deep space head and neck infections. Oral Surg Oral Med Oral Pathol Oral Radiol Endod 2010;110: 151-6.

32. Pericàs JM, Nathavitharana R, Garcia-de-la-Mària C, Falces C, 
Ambrosioni J, Almela M, et al. Endocarditis caused by highly penicillin-resistant viridans group streptococci: still room for vancomycin-based regimens. Antimicrob Agents Chemother 2019;63:e00516-19.

33. Eshetie S, Unakal C, Gelaw A, Ayelign B, Endris M, Moges F. Multidrug resistant and carbapenemase producing Enterobacteriaceae among patients with urinary tract infection at referral Hospital, Northwest Ethiopia. Antimicrob Resist Infect Control 2015;4:1-8

34. Raji MA, Jamal W, Ojemhen O, Rotimi VO. Point-surveillance of antibiotic resistance in Enterobacteriaceae isolates from patients in a Lagos Teaching Hospital, Nigeria. J Infect Public Health 2013:6:431-7.

35. Ullah W, Qasim M, Rahman H, Bari F, Khan S, Rehman ZU, et al. Multi drug resistant Pseudomonas aeruginosa pathogen burden and associated antibiogram in a tertiary care hospital of Pakistan. Microb Pathog 2016;97:209-12.
36. Godebo G, Kibru G, Tassew H. Multidrug-resistant bacterial isolates in infected wounds at Jimma University Specialized Hospital, Ethiopia. Ann Clin Microbiol Antimicrob 2013;12:1-7.

37. Moges F, Endris M, Belyhun Y, Worku W. Isolation and characterization of multiple drug resistance bacterial pathogens from waste water in hospital and non-hospital environments, Northwest Ethiopia. BMC Res Notes 2014;7: $1-6$.

38. Khan HA, Baig FK, Mehboob R. Nosocomial infections: epidemiology, prevention, control and surveillance. Asian Pac J Trop Biomed 2017;7:478-82.

39. Farooq L, Memon Z, Ismail MO, Sadiq S. Frequency and antibiogram of multi-drug resistant Pseudomonas aeruginosa in a Tertiary Care Hospital of Pakistan. Pak J Med Sci 2019;35: 1622-6. 